INVESTIGACIÓN

https://doi.org/10.15198/seeci. 2020.52.1-15

Recibido: 18/07/2019 --- Aceptado: 10/09/2019 --- Publicado: 15/07/2020

\title{
LAS TIC COMO HERRAMIENTA DE INVESTIGACIÓN: ANALIZANDO LAS TÉCNICAS DE PROGRAMACIÓN NEUROLINGUÍSTICA
}

\section{ICT AS A RESEARCH TOOL: ANALYZING NEUROLINGUISTIC PROGRAMMING TECHNIQUES}

Marta Cabaleiro Pedrero ${ }^{1}$. ESERP Business \& Law School, centro adscrito a la Universidad Rey Juan Carlos. Madrid, España.

mcabaleirop@gmail.com

José Rodríguez Terceño². ESERP Business \& Law School, centro adscrito a la Universidad Rey Juan Carlos. Madrid, España.

joserodriguez@eserp.com

José Ramón Sarmiento Guede ${ }^{3}$. Universidad Rey Juan Carlos. Madrid, España.

Joseramon.sarmiento@urjc.es

\section{RESUMEN}

En el presente trabajo de investigación vamos a analizar en profundidad las herramientas características de Programación Neurolingüística y cuestiones íntimamente relacionadas y vinculadas a su entorno, desarrollo, uso y percepción social, con el objetivo último de demostrar o refutar su veracidad respecto a los diferentes tipos de aprendizaje que abarca esta disciplina y a los que afecta indirectamente, así como la idea misma de que la dirección de la mirada (la dirección en la que la persona enfoca los ojos mientras habla con otra, señalando a zonas del cerebro con funciones específicas conocidas) de una persona supone o no un indicativo fiable sobre si alguien miente o dice la verdad. El contenido del trabajo consiste en haber puesto en práctica esta hipótesis y en un esfuerzo por probar sus resultados. De este modo tenemos el objetivo último de alcanzar una conclusión sobre el hecho de si son comprobables o no, refutables o no las herramientas de

\footnotetext{
${ }^{1}$ Marta Cabaleiro Pedrero: graduada en Marketing por ESERP Business \& Law School, centro universitario adscrito a la Universidad Rey Juan Carlos de Madrid.

mcabaleirop@gmail.com

2José Rodríguez Terceño: profesor docente y coordinador académico en ESERP Business \& Law School, centro universitario adscrito a la Universidad Rey Juan Carlos de Madrid. joserodriguez@eserp.com

3José Ramón Sarmiento Guede: Profesor en la Universidad Rey Juan Carlos de Madrid. joseramon.sarmiento@urc.es.
} 
Programación Neurolingüística y sus elementos asociados; ya que ésta actualmente no está fundamentada ni demostrada con herramientas, técnicas y tecnologías que sean consideradas medibles a diferencia de otras herramientas que si han contado con el beneficio de esta comprobación, como por ejemplo el Eye Tracking.

PALABRAS CLAVE: programación neurolingüística - PNL - análisis - herramientas de programación neurolingüística - movimiento ocular - tipo de aprendizaje detector de mentiras.

\section{ABSTRACT}

In this research paper, we are going to analyze in depth the characteristic neurolinguistic programming tools and issues closely related and linked to their environment, development, use and social awareness, with the final aim to prove or disprove their veracity regarding the different types of learning embraced and indirectly affected by this discipline, as well as the idea itself that the direction of one's look (the direction in which the person focuses their eyes while talking with another, pointing to areas of the brain with specific known functions) either entails or not a reliable indication of whether someone is lying or telling the truth. The content of this paper consists in having put this hypothesis into practice and in an effort to test its results. Thus we have the ultimate goal of reaching a conclusion on the fact of whether neurolinguistic programming tools and their related elements are testable or not, refutable or not; as it is currently not substantiated or proven with tools, techniques and technologies that are considered measurable unlike other tools that have had the benefit of this test, such as, for example, Eye Tracking.

KEYWORDS: neurolinguistic programming - NLP - analysis - neurolinguistic programming tools - eye movement - learning type - lie detector.

\section{AS TIC COMO FERRAMENTA DE PESQUISA: ANALISANDO AS TÉCNICAS DE PROGRAMAÇÃO}

\section{RESUMO}

No presente trabalho de pesquisa vamos analisar em profundidade as ferramentas características da Programação Neurolinguística e as questões intimamente ligadas e vinculadas ao seu entorno, desenvolvimento, uso e percepção social, com o objetivo final de demonstrar ou refutar a sua veracidade respeito aos diferentes tipos de aprendizado que esta disciplina abarca e aos que são afetados indiretamente, assim como a própria ideia de que a direção do olhar (a direção na que a pessoa foca os olhos enquanto fala com outra, sinalizando as zonas do cérebro com funções específicas conhecidas) de uma pessoa supõe ou não um indicativo confiável sobre se alguém mente o diz a verdade. $O$ conteúdo do trabalho se baseia na colocação em prática desta hipótese e no esforço por provar os seus resultados. Deste modo temos como objetivo final conseguir uma conclusão sobre o fato de serem comprováveis ou não, refutáveis ou não às ferramentas de Programação Neurolinguística e os seus elementos associados; já que a mesma atualmente não 
está fundamentada nem demonstrada com ferramentas, técnicas e tecnologias que sejam consideradas mensuráveis a diferença de outras ferramentas que contam com o benefício da comprovação, como, por exemplo o Eye Tracking.

PALAVRAS CHAVE: programação neurolingüística - PNL - análise - ferramentas de programação neurolinguística - movimento ocular - tipo de aprendizado - detetor de mentiras.

\section{Cómo citar el artículo:}

Cabaleiro Pedrero, M.; Rodríguez Terceño, J. y Sarmiento Guede, J. R. (2020). Las TIC como herramienta de investigación: analizando las técnicas de Programación Neurolingüística. [ICT as a research tool: analyzing Neurolinguistic Programming techniques]. Revista de Comunicación de la SEECI, (52), 1-15. doi: http://doi.org/10.15198/seeci.2020.52.1-15

Recuperado de http://www.seeci.net/revista/index.php/seeci/article/view/611

\section{INTRODUCCIÓN}

Todos alguna vez nos hemos preguntado si la persona que estaba delante nuestra contándonos cualquier cosa estaba mintiendo o nos estaba diciendo la verdad. Una pregunta si cabe más importante con las crecientes facilidades de comunicación, tal como apuntan Lavilla Muñoz y Mesonero Izquierdo (2010) y amplían Álvarez Flóres (2017) y Vázquez-Herrero, Negreira-Rey y Pereira-Fariña (2017). Mirar a los ojos fijamente o deslizar la mirada a la izquierda, son gestos que se consideran detectores de mentiras y que la mayoría da por hecho que son ciertos.

Aprender a mentir es algo complejo que puede llevar tiempo dentro del papel dinámico del lenguaje en la sociedad (Manzano Díaz, Bravo Alvarado y García Lebroc, 2019) pero hay herramientas que nos ayudarán a darnos cuenta de si alguien nos está mintiendo incrementando el valor de la comunicación personal en un contexto de mentira digital generalizada como el descrito por Bustos, De Santiago et al. (2019) y antes por Timoteo Álvarez (2007). Una de esas herramientas es la Programación Neurolingüística (a partir ahora, PNL). La PNL puede ayudar a crear el ambiente o el entorno físico adecuado, haciendo que la otra persona se sienta más cómoda, y lo más importante enseñarnos a proyectar con los gestos, no con las palabras, una imagen predeterminada de forma consciente. De modo que la PNL promete que puede hacer que una persona influya sobre otra si toma ciertas actitudes y posiciones que le ayuden a empatizar.

La PNL se basa en que, si analizamos de forma algo más detallada la comunicación verbal y no verbal, y usando ésta información de forma correcta, nos puede ayudar a persuadir a la otra persona de la manera que sea -en la línea de lo aportado por Blanco Mallada (2009)-. Esto es factible para algunos hasta el punto de ver la Programación Neurolingüística como una ciencia (Tal como definen ciencia 
Mestres Naval y Vives-Rego, 2016) y no sólo una herramienta que facilite la comunicación.

Investigaremos sobre los diferentes tipos de aprendizaje que explica esta disciplina, y cómo la dirección de la mirada de una persona en teoría dictamina si alguien miente o dice la verdad. Es decir, pondremos en práctica esta hipótesis y probaremos sus resultados.

El propósito de este trabajo es estudiar y evaluar si realmente la PNL tiene una base aparentemente científica que se pueda corroborar. Intentar estudiar si la dirección de la mirada tiene alguna relación con decir la verdad o no.

Para consolidar el trabajo estudiaremos diferentes fuentes y estudios que traten este tema en profundidad y llevaremos la teoría de la PNL a la práctica mediante un experimento, para así poder medir su fiabilidad. De este modo alcanzaremos una conclusión sobre si son comprobables o refutables las herramientas de PNL.

A continuación, procederemos a especificar el objeto y los objetivos de la investigación.

\section{OBJETIVOS}

Como objetivo general, estudiaremos a fondo la PNL, pretendiendo llegar a una conclusión relevante sobre su base científica o experiencial/experimental y medir la fiabilidad de las herramientas de la PNL.

Respecto a los objetivos específicos:

- Analizar los distintos campos en los que se ha aplicado o se aplica.

- Llevar a cabo una investigación sobre las herramientas que se usan para medir la capacidad de la PNL dependiendo del campo en el que se encuentra.

- Ahondar en sus aplicaciones en una entrevista personal.

- Las diferentes direcciones visuales para detectar si una persona dice la verdad o miente y así más adelante poder llevar todo lo analizado a la práctica.

Una vez alcanzados los objetivos mencionados pretendemos obtener unas conclusiones que, según su pertinencia sobre lo analizado, nos permitirán comprobar si estas herramientas son o no correctas, en términos de fiabilidad, comprobación /corroboración o refutación.

\section{METODOLOGÍA}

La metodología que llevaremos a cabo para poder demostrar la eficacia de la Programación Neurolingüística será, por un lado, cualitativa, ya que se llevará a cabo una entrevista personal (Anexos, en el punto 8) estructurada en relación a qué método de aprendizaje es predominante en esa persona (lo explicaremos a continuación) y también será experiencial, ya que se llevará a cabo un experimento 
que intenta probar la veracidad y fiabilidad, en los términos ya mencionados de las herramientas de la PNL que se utilizan en diferentes ámbitos.

El método consistirá en utilizar como soporte visual una cámara de video que estará colocada frente a los sujetos en un primer plano con la que grabaremos su movimiento ocular al responder a las preguntas de la entrevista para averiguar si los hechos que dicen son verdaderos o no, es decir, poder corroborar si realmente utilizando herramientas de la PNL podemos saber si mienten o dicen la verdad. En otras palabras, queremos comprobar si podemos deducir si mienten o dicen la verdad según sus movimientos oculares, o si, por el contrario, no hay ninguna relación entre el movimiento ocular y la veracidad de sus palabras. De este modo, al dejarlo grabado, quedará constancia de los movimientos oculares y nos permitirá hacer revisiones de la dirección en la que se dirige la mirada en cada momento de la sesión.

Este sistema será suficientemente completo y cuenta con las características necesarias como para poder detectar, obtener y registrar los cambios del movimiento ocular. Sería algo parecido a usar el Eye-Tracking (salvando las diferencias y equipamientos, por supuesto) para medir el movimiento y la posición del ojo en relación a la cabeza. Es utilizado en campos de investigación como: neurociencia, psicología, marketing y publicidad, informática, diseño de productos... (Hassan Montero y Herrero Solana, octubre 2007).

El Eye-Tracking es una herramienta que, al fin y al cabo, tiene unos resultados demostrables y medibles que contienen datos estadísticos, cuantificables, en definitiva. Sin embargo, a diferencia de esta técnica, en la PNL no hay ninguna herramienta como tal que pueda medir su veracidad. Es decir, que todo lo que se ha estudiado o descrito sobre cómo la dirección de la mirada demuestra una mentira, no está fundamentado ni demostrado, con herramientas, técnicas y tecnologías que sean medibles. Y esto es lo que nos ha dado pie a comenzar la investigación e intentar corroborar si todo lo que se ha escrito está fundamentado o no científicamente.

Para el experimento escogeremos dos sujetos de referencia, uno procederá a contestar a las últimas preguntas más extensas (de la pregunta 19-21) diciendo únicamente la verdad, y el otro hará exactamente lo contrario, contestando con hechos que no son ciertos. Ambos dirigirán la mirada hacia un lado u otro (dependiendo de si mienten o no) de forma intencionada para poder compararlos con el resto, ya que ellos se limitarán a escenificar la teoría de la PNL. Una vez realizadas estas dos grabaciones seleccionaremos a otras diez personas que responderán a las preguntas para poder analizar sus movimientos oculares y posteriormente poderlos comparar con los de referencia. De las diez, cinco contestarán diciendo la verdad y las otras cinco mintiendo. De este modo al contrastar el movimiento ocular se podrá deducir que: si la persona que participa, repite el patrón del sujeto de referencia que miente, éste también lo estará haciendo y viceversa.

Además de llevar a cabo las comparaciones entre los diez sujetos y los dos de referencia, tendremos que tener en cuenta que también es importante conocer qué 
tipo de personalidad o estilo de aprendizaje tienen según la PNL (visual, auditivo o kinestésico). Según ésta hay hasta seis direcciones visuales, y cada una de ellas indica algo diferente. Básicamente dependiendo de hacia dónde dirija la mirada la persona, puede estar creando (mira hacia la izquierda) o recordando (mira hacia la derecha). A su vez estas dos direcciones se clasifican según el tipo de persona que eres: visual, auditivo o kinestésico.

Llegando al tema que nos interesa, para saber cómo detectar una mentira hay que tener en cuenta que cuando mientes no te basas en un recuerdo, sino que la mente lo va construyendo. De modo que cuando alguien miente la mirada se dirige siempre hacia la izquierda (Migallón, 2011).

\section{DISCUSIÓN}

Lo primero, recordaremos brevemente la metodología a utilizar para corroborar la eficacia de las herramientas de la PNL. Se trata de una entrevista personal estructurada (Anexos, en el punto 8) que consta de veintiuna preguntas que están divididas en varios grupos. Las seis primeras tratan de forzar la utilización del canal visual, las seis siguientes del canal auditivo y las siguientes seis que siguen a éstas, del kinestésico. Dentro de cada grupo de seis, estarán divididas en dos partes: una (correspondiente a las tres primeras) que tratarán de que el entrevistado conteste recordando y por tanto de forma verídica (su mirada debe dirigirse hacia la derecha), y las tres siguientes tratarán de que use su imaginación (su mirada debe dirigirse hacia la izquierda). De modo que el esquema de la entrevista es el siguiente:

\begin{tabular}{|c|c|}
\hline Visual & $\begin{array}{ll}\text { 1. } & \text { Recuerdo } \\
\text { 2. } & \text { Recuerdo } \\
\text { 3. Recuerdo } \\
\text { 4. Imaginación } \\
\text { 5. Imaginación } \\
\text { 6. Imaginación }\end{array}$ \\
\hline Auditivo & $\begin{array}{l}\text { 7. Recuerdo } \\
\text { 8. Recuerdo } \\
\text { 9. Recuerdo } \\
\text { 10. Imaginación } \\
\text { 11. Imaginación } \\
\text { 12. Imaginación }\end{array}$ \\
\hline Kinestésico & $\begin{array}{l}\text { 13. Recuerdo } \\
\text { 14. Recuerdo } \\
\text { 15. Recuerdo } \\
\text { 16. Imaginación o diálogo interno } \\
\text { 17. Imaginación o diálogo interno } \\
\text { 18. Imaginación o diálogo interno }\end{array}$ \\
\hline
\end{tabular}

Esquema 1: Significado/organización de la entrevista.

Fuente: elaboración propia. 
Con estas dieciocho preguntas probaremos si: con las preguntas visuales, las personas entrevistadas realmente dirigen su mirada hacia arriba; con las auditivas, su campo de visión se encuentra en la zona central; y con las kinestésicas si la dirección de la mirada se dirige hacia abajo, tal y como afirma la PNL.

Respecto a las tres últimas preguntas (de la dieciocho en adelante), al tratarse de preguntas más extensas, analizaremos su movimiento ocular respecto a si dirige su mirada hacia la izquierda, según se ve en cámara, o a la derecha. De modo que, si mira hacia la izquierda significa que está usando la imaginación y por lo tanto miente; y si por el contrario lo hace hacia la derecha, es que está recordando algún hecho, y por lo tanto dice la verdad. Al ser preguntas abiertas y más extensas dejaremos que el entrevistado pueda desarrollar su tipo de canal predominante sin condicionamientos.

Algo a mencionar es que, las personas no miran únicamente todo el rato hacia una misma dirección, así que se analizará hacia dónde tiende a dirigirse la mirada dependiendo de la pregunta.

La entrevista será realizada a doce personas, de las cuáles dos serán los sujetos de referencia. Estas dos personas llevarán a cabo la teoría de la PNL en cuanto al movimiento ocular que deberían realizar según el tipo de pregunta de la que se trata (visual hacia arriba, auditiva en el centro y kinestésica hacia abajo), respondiendo a las preguntas de la forma más natural posible. También se verá reflejado que: el sujeto de referencia 1 en las tres últimas preguntas responderá dirigiendo la mirada hacia la derecha, por lo que simula que dice la verdad en todo momento; y el sujeto de referencia 2 tenderá a hacerlo hacia la izquierda reflejando el efecto contrario. Así nos ayudarán a comparar sus movimientos que representan la teoría que afirma la PNL (tanto en el tipo de canal que se utiliza según qué recuerdos o tipo de imaginación utilice, y el cómo detectar una mentira según la dirección de la mirada) con los del resto.

De las diez personas restantes, cabe decir que, cinco contestarán diciendo la verdad y las otras cinco mintiendo, y así podremos comparar sus respuestas con las de los sujetos de referencia. Por tanto, al contrastar el movimiento ocular se podrá deducir que: si la persona que participa repite el patrón del sujeto de referencia 2, que es el sujeto de referencia que miente, éste también lo estará haciendo y viceversa. Destacando que la fuente de elaboración de las preguntas es propia.

El perfil de las personas que hemos seleccionado para el experimento es muy amplio y al azar, puesto que variables como el sexo o la edad no modifican los resultados. Esto se ha realizado así ya que la PNL afirma que, las microexpresiones anteriormente mencionadas, son universales y no dependen de la cultura o país. Por lo tanto, no hay variables socioeconómicas, ni geográficas, ni psicográficas o conductuales.

Aun así, respecto a las variables sociodemográficas podemos decir que: de las doce personas escogidas cuatro son hombres y ocho son mujeres (que corresponde a un $33,33 \%$ y un $66,66 \%$ respectivamente); que el rango de edad que comprende el experimento es desde los 21 a los 63 años, teniendo en su mayoría entre 21 y 25 
años; y que el lugar de residencia en todos de los entrevistados es Madrid, a excepción de dos personas.

A continuación, pasaremos a describir a los sujetos del estudio y cuáles fueron sus respuestas en relación a su movimiento ocular.

\subsection{Los sujetos de referencia del estudio}

Los sujetos de referencia son dos personas escogidas al azar que se encargarán de escenificar las acciones que, supuestamente deberían realizar el resto de sujetos al contestar a las preguntas.

Sus acciones quedarán divididas, al igual que la entrevista, en dos partes. La primera, está compuesta por las primeras dieciocho preguntas, en la que deberán representar dos cosas. Por un lado, el movimiento ocular en relación al tipo de canal que deberían estar utilizando (visual, auditivo o kinestésico) dependiendo del tipo de pregunta. Y por otro la dirección de la mirada en relación a si se trata de un recuerdo $o$ un hecho imaginativo.

De manera que el movimiento ocular en la primera parte de la entrevista debería ser para ambos sujetos la siguiente:

Tabla 1. Movimiento ocular de los sujetos de referencia.

\begin{tabular}{|c|c|c|}
\hline & RECUERDO & IMAGINACIÓN \\
\hline $\begin{array}{c}\text { VISUAL } \\
\text { (arriba) }\end{array}$ & $\begin{array}{c}\text { Mirada hacia arriba a la } \\
\text { derecha. }\end{array}$ & $\begin{array}{c}\text { Mirada hacia arriba a la } \\
\text { izquierda. }\end{array}$ \\
\hline $\begin{array}{c}\text { AUDITIVO } \\
\text { (zona central) }\end{array}$ & Mirada al centro a la derecha. & $\begin{array}{c}\text { Mirada al centro a la } \\
\text { izquierda. }\end{array}$ \\
\hline $\begin{array}{c}\text { KINESTÉSICO } \\
\text { (abajo) }\end{array}$ & $\begin{array}{c}\text { Mirada hacia abajo a la } \\
\text { izquierda. }\end{array}$ & $\begin{array}{c}\text { Mirada hacia abajo a la } \\
\text { derecha (diálogo interno) }\end{array}$ \\
\hline
\end{tabular}

Fuente: elaboración propia.

La segunda parte de la entrevista que abarca las tres últimas preguntas como ya hemos mencionado el sujeto de referencia 1 responderá dirigiendo la mirada hacia la derecha, por lo que simula que dice la verdad en todo momento por usar recuerdos de su memoria; y el sujeto de referencia 2 tenderá a hacerlo hacia la izquierda reflejando el efecto contrario por usar la imaginación al elaborar una respuesta.

Sabiendo esto vamos a pasar a analizar al resto de sujetos que han formado parte del estudio, los diez restantes, para poder extraer conclusiones que nos ayuden a comprobar la fiabilidad de las herramientas de PNL.

Revista de Comunicación de la SEECI. 15 julio, 2020 / 15 noviembre, 2020, nº 52, 1-15 


\subsection{El resto de sujetos del estudio}

Al igual que en punto anterior vamos a estructurar las entrevistas en dos partes. Empezaremos por el movimiento ocular en relación al canal que utiliza para expresarse (visual, auditivo o kinestésico) y posteriormente a la comprobación de la veracidad en sus contestaciones según si su movimiento ocular tiende hacia la izquierda (miente en sus respuestas) o derecha (cuyas respuestas serán ciertas). De este modo con la entrevista forzamos a la utilización de todos los canales y dejamos las últimas preguntas abiertas para que "desarrolle" su tipo de canal de aprendizaje predominante sin condicionamientos.

\subsubsection{El movimiento ocular y su canal sensorial}

Con las preguntas que hemos realizado hemos buscado contestaciones basadas en recuerdos visuales, auditivos y kinestésicos. De modo que si la pregunta evoca a utilizar el canal visual la mirada debe dirigirse hacia arriba, si es auditiva en la zona central y si es kinestésico hacia abajo. Por otro lado también, si sugiere un recuerdo su mirada irá hacia la derecha, y si es algo imaginario hacia la izquierda. Además de tener esto en cuenta, debemos destacar que para obtener resultados más claros se compararán los movimientos oculares con los sujetos de referencia sin importar con cuál.

Para que la compresión sea más clara éste es un breve esquema de cuál sería el movimiento ocular ${ }^{4}$ adecuado de la mirada en relación a lo explicado. De modo que:

Preguntas de la 1-3 (visual, recuerdo): mirada hacia arriba y a la derecha.

Preguntas de la 4-6 (visual, imaginación): mirada hacia arriba y a la izquierda.

Preguntas de la 7-9 (auditivo, recuerdo): mirada al centro y a la derecha.

Preguntas de la 10-12 (auditivo, imaginación): mirada al centro y a la izquierda.

Preguntas de la 13-15 (kinestésico, recuerdo): mirada hacia abajo y a la izquierda. Preguntas de la 16-18 (kinestésico, diálogo interno): mirada hacia abajo y a la derecha.

Recordamos que para una mejor comprensión de los puntos posteriores es recomendable revisar el esquema 1 (dentro del punto 4).

Después de analizar el movimiento ocular sujeto por sujeto pudimos observar que todos los sujetos presentaron una clara predilección por el uso del canal visual, puesto que la mayor parte del tiempo dirigieron su mirada hacia arriba. Siendo éste realizado a la perfección por dos sujetos, no solo en dirigir la mirada hacia arriba sino también en el sentido de izquierda y derecha.

${ }^{4}$ El movimiento ocular se describe según la percepción del observador. De modo que, cuando se habla de que el sujeto mira hacia la izquierda, es desde el punto de vista del entrevistador y no del entrevistado. 
Podemos destacar que el movimiento ocular que más veces ha coincidido con lo estipulado por la PNL es el correspondiente al canal kinestésico cuando se trata de un diálogo interno (abajo a la derecha).

Cabe mencionar que independientemente del canal utilizado varios sujetos tuvieron predilección por dirigir su mirada hacia la izquierda, algo que será relevante en la segunda parte del experimento.

También debemos tener en cuenta que si un sujeto repite un movimiento muy definido en algún momento coincidirá con lo estipulado por la PNL por probabilidad. De modo que no sería del todo fiable considerar ese movimiento como un acierto: la lectura de gestos no llega a la fiabilidad de la comprensión de textos, tal como la entiende Odalis Lorié (2015).

\subsubsection{El movimiento ocular como detector de mentiras}

En este punto nos centraremos en analizar el movimiento ocular únicamente en relación a la dirección de la mirada entre la izquierda o la derecha, sabiendo que, si la mirada centra su atención principalmente en la derecha el sujeto estará diciendo la verdad al tratarse de un recuerdo, y si por el contrario centra la atención en la izquierda el sujeto miente.

Los sujetos respondieron a las tres últimas preguntas de la entrevista de forma abierta y sin condicionamientos, siendo estas: qué hizo el fin de semana pasado, qué haría si le tocase la lotería, y cuénteme su último viaje. La mitad de los sujetos respondieron de forma sincera, por lo que éstos se compararán con las acciones del sujeto de referencia 1 , y la otra mitad que no lo hicieron tendrán de ejemplo al sujeto de referencia 2 .

Lo primero que haremos será explicar los movimientos oculares que han llevado a cabo los sujetos, es decir tendremos en cuenta el número de veces que su mirada se dirige a la izquierda cuyo significado es que miente (como el sujeto de referencia 2), o a la derecha el cual es decir la verdad (como el sujeto de referencia 1). También tendremos en cuenta otros indicadores que pueden mostrar si alguien miente, como: picor en la boca, sudor, ojos fijos, palidez, justificarse en exceso, bajar la voz, tragar saliva o sentir alivio al terminar de responder.

Hemos realizado un cuadro resumen de todos los sujetos, el número de veces que mira hacia la izquierda y a la derecha, los indicadores, la interpretación según la PNL y la respuesta real que dieron los entrevistados. De este modo podremos comparar de forma rápida si es fiable esta herramienta de PNL. Dando como resultado la siguiente tabla: 
Cabaleiro Pedrero, M.; Rodríguez Terceño, J. y Sarmiento Guede, J. R. Las TIC como

herramienta de investigación: analizando las técnicas de Programación Neurolingüística

Tabla 2. Segunda parte de la entrevista, detectar mentiras.

\begin{tabular}{|c|c|c|c|c|c|}
\hline & $\begin{array}{c}\text { Mirada a la } \\
\text { izquierda }\end{array}$ & $\begin{array}{c}\text { Mirada a la } \\
\text { derecha }\end{array}$ & $\begin{array}{c}\text { Otros } \\
\text { indicadores }\end{array}$ & $\begin{array}{l}\text { Interpretación } \\
\text { según PNL }\end{array}$ & $\begin{array}{c}\text { Respuesta } \\
\text { real }\end{array}$ \\
\hline Sujeto 1 & 14 & 15 & Boca seca & Indefinido & Verdad \\
\hline Sujeto 2 & 2 & 7 & & Verdad & Miente \\
\hline Sujeto 3 & 8 & 6 & $\begin{array}{l}\text { Repetir } \\
\text { pregunta }\end{array}$ & Miente & Miente \\
\hline Sujeto 4 & 18 & 16 & & Miente & Verdad \\
\hline Sujeto 5 & 14 & 8 & $\begin{array}{c}\text { Boca seca. } \\
\text { Morderse labios }\end{array}$ & Miente & Verdad \\
\hline Sujeto 6 & 11 & 9 & & Miente & Miente \\
\hline Sujeto 7 & 19 & 15 & $\begin{array}{c}\text { Justificarse en } \\
\text { exceso }\end{array}$ & Miente & Verdad \\
\hline Sujeto 8 & 14 & 6 & & Miente & Miente \\
\hline Sujeto 9 & 13 & 20 & $\begin{array}{l}\text { Repetir } \\
\text { pregunta }\end{array}$ & Verdad & Miente \\
\hline Sujeto 10 & 1 & 18 & $\begin{array}{c}\text { Justificarse en } \\
\text { exceso. } \\
\text { Tragar saliva }\end{array}$ & Verdad & Verdad \\
\hline
\end{tabular}

Fuente: elaboración propia.

Como se puede observar en la mayoría de los casos la PNL no ha resultado fiable en cuanto a la veracidad de las palabras de los entrevistados. Aun así cuenta con un $44 \%{ }^{5}$ de acierto, lo que le da cierta credibilidad, o al menos en un principio.

Consideramos que tener en cuenta si ha dirigido la mirada más veces hacia la izquierda o a la derecha no es del todo fiable si no se contempla el movimiento ocular mencionado en tablas anteriores. Por ejemplo, hay muchos sujetos que han presentado una clara tendencia hacia la izquierda (en su mayoría mediante el canal visual) y los sujetos han respondido con sinceridad. Por lo que, según la PNL todos los que han tenido esa predilección por el lado izquierdo han estado mintiendo en todo momento.

\section{CONCLUSIONES Y FUTURAS APLICACIONES}

Así pues, la PNL es entendida como una pseudociencia (dícese, que huye de la pruebas y basa su credibilidad en visiones adulteradas del éxito de sus propias predicciones, acorde a Gutiérrez Muñoz, 2007), cuyas herramientas no han sido probadas ni demostradas científicamente. Con nuestra investigación y mediante la realización de un experimento, llevado a cabo para conocer si la habilidad de la PNL para detectar si una persona miente es fiable, hemos podido corroborar la hipótesis inicial. La PNL no es una herramienta cien por cien fiable.

Hemos observado cómo no hay una relación exacta entre la dirección de la mirada y el tipo de canal de aprendizaje que predomina en una persona (visual, auditivo o

\footnotetext{
${ }^{5}$ El porcentaje real de un $44,44 \%$ periódico. Está realizado el porcentaje sobre nueve sujetos, no diez
} debido a que el sujeto 1 no tiene unos resultados concluyentes. 
kinestésico). Es decir, mirar hacia arriba cuando se está reflexionando sobre qué responder a una pregunta, no implica que su canal de percepción sea principalmente visual. De la misma manera que esto ocurre, aunque de forma algo más acertada, mirar hacia la izquierda no supone que la persona esté mintiendo de forma constante. Sino que puede ser una costumbre o una manía.

También hemos analizado cómo la PNL se aplica en campos muy dispares como: neurología, educación o en una organización empresarial; y que hay cantidad de herramientas o consejos que nos ayudan a emplear la PNL de la mejor manera posible. Por ejemplo, adoptar la misma postura que otra persona, respirar al unísono o mover las manos de una manera u otra, puede ser relevante al mantener una conversación cara a cara. Al fin y al cabo, somos personas que, en mayor o menor medida empatizamos con otras, y si uno opta por una postura a la defensiva la otra persona acabará optando por hacer lo mismo.

Las herramientas de PNL, en lo referente al movimiento ocular, al no ser del todo fiables no serían recomendables aplicarlas en ciertos ámbitos. Consideramos que en campos donde su relevancia es importante, como emplearlo en un proceso de selección o como detector de mentiras ante un acto de delincuencia, no es lo adecuado.

Nuestro objeto de estudio, centrado en la aplicación de la PNL en una entrevista personal, nos ha ayudado a poner en práctica las herramientas en relación al movimiento ocular y a pesar de que el resultado no ha sido favorable del todo hemos de señalar varios puntos.

La PNL es mucho más amplia que el movimiento ocular pues tiene en cuenta ciertos indicadores, para averiguar si alguien miente, y éstos están íntimamente relacionados con el lenguaje no verbal o la sinergología. Entendiéndose por sinergología la "relación entre el lenguaje del cuerpo y las palabras" (Turchet, 2010). Es decir, trata de analizar los movimientos corporales que realiza una persona de forma inconsciente.

Al centrarnos únicamente en el movimiento ocular y su significado, es posible que no hayamos abarcado lo suficiente como para que las herramientas de PNL tengan mayor validez. Así pues, consideramos posteriormente llevar a cabo una investigación experiencial parecida a la realizada, que abarque no sólo la mirada sino también los microgestos o la forma de expresarse verbalmente, es decir, introduciremos otras variables o factores que la comunicación no verbal tiene en consideración y que hemos podido destacar en nuestra investigación.

\section{REFERENCIAS}

Álvarez-Flores, E. P.; Núñez-Gómez, P. \& Rodríguez Crespo, C. (2017). E-skills acquisition and deficiencies at the university in the context of the digital economy. Revista Latina de Comunicación Social, (72), 540-559. doi: http://dx.doi.org/10.4185/RLCS-2017-1178 
Bustos Martínez, L.; De Santiago Ortega, P. P.; Martínez Miró, M. A. y Rengifo Hidalgo, M. S. (2019). Discursos de odio: una epidemia que se propaga en la red. Estado de la cuestión sobre el racismo y la xenofobia en las redes sociales. Revista Mediaciones Sociales, (18), 25-42. doi: http://dx.doi.org/10.5209/MESO.64527

Blanco Mallada, L. (2009). La gestualidad: significación y estética del rostro y la expresión corporal. Revista Vivat Academia, (105), 40-58. Recuperado de http://www.vivatacademia.net/index.php/vivat/article/view/256/230

Gutierrez Muñoz, J. (2007). Ciencia frente a pseudociencia. Revista Vivat Academia (90), 1-34. doi: http://dx.doi.org/10.15178/va.2007.90.1-34

Hassan Montero, Y., y Herrero Solana, V. (2007). Eye-Tracking en Interacción Persona-Ordenador. No Solo Usabilidad, (6). Recuperado de http://www.nosolousabilidad.com/articulos/eye-tracking.htm

Lavilla Muñoz, D., y Mesonero Izquierdo, R. (2010). Realidad y ficción. Desde la autopercepción a la sociabilización tecnológica: discurrir histórico. Revista de la SEECI, (22), 16-29. doi: https://doi.org/10.15198/seeci.2010.22.16-29

Lorié González, O. (2015). Modelo didáctico de la comprensión de textos escritos desde una perspectiva lingüístico-pedagógica. Revista Inclusiones, 2(3), 76-87.

Recuperado de

http://www.archivosrevistainclusiones.com/gallery/6\%20oficial\%20articulo\%20jul \%20sep\%20\%20\%202015\%20rev\%20inc.pdf

Manzano Díaz, M.; Bravo Alvarado, R. N., y García Lebroc, L. A. (2019). Las competencias comunicativas en la formación permanente: discursos y realidades. Revista Inclusiones, 6(2), 87-106. Recuperado de http://www.archivosrevistainclusiones.com/gallery/5\%20vol\%206\%20num\%202 \%202019abriljunio19incl.pdf

Mestres Naval, F. y Vives-Rodrigo, J. (2016). Justicia y Ciencia: avanzando juntas para construir un mundo mejor. Revista inclusiones, 4(2), 10-27. Recuperado de http://www.archivosrevistainclusiones.com/gallery/1\%20oficial\%202016\%20abr\% 20jun\%20rev\%20inc.pdf

Migallón, I. (2011). Cómo detectar mentiras según la mirada. Psicocode. Recuperado de https://psicocode.com/social/como-detectar-mentiras-segun-la-mirada/

Timoteo Álvarez, J. (2007). Neurocomunicación. Propuesta para una revisión de los fundamentos teóricos de la comunicación y sus aplicaciones industriales y sociales. Mediaciones Sociales, (1), 355-386. Recuperado de https://revistas.ucm.es/index.php/MESO/article/view/MESO0707110355A/21499

Turchet P. (2010). El lenguaje del cuerpo: conozca a su interlocutor a través de sus gestos y posturas. Editorial Ediciones Mensajero, S.A. 
Vázquez-Herrero, J.; Negreira-Rey, M. C., \& Pereira-Fariña, X. (2017). Interactive documentary contributions to the renewal of journalistic narratives: realities and challenges. Revista Latina de Comunicación Social, (72), 397-414. doi: http://dx.doi.org/10.4185/RLCS-2017-1171en

\section{AUTORES:}

\section{Marta Cabaleiro Pedrero}

Graduada en Marketing por ESERP Business \& Law School y por la Universidad Rey Juan Carlos de Madrid, estudiante de post-grado especializado en marketing digital, presenta con esta investigación su primera aportación divulgativa fruto del esfuerzo empleado en la materialización de su trabajo fin de carrera.

mcabaleirop@gmail.com

\section{José Rodríguez Terceño}

Licenciado en Comunicación Audiovisual y Doctor en Ciencias de la Información por la Universidad Complutense de Madrid. Miembro del Grupo de Investigación Validado Complutense Concilium, ha participado en diversas publicaciones colectivas centradas en la séptima arte, periodismo y las relaciones públicas.

joserodriguez@eserp.com

Orcid ID: https://orcid.org/0000-0003-2859-4181

\section{José Ramón Sarmiento Guede}

Profesor en la Universidad Rey Juan Carlos y colaborador docente en post-grado en otros centros. Ha trabajado durante un año en la agencia de marketing Gestión de Mecenazgo en la que desarrollaba campañas de comunicación para Central Lechera Asturiana. Además, ha trabajo en el Departamento de Relaciones Externas y de Marketing de IFEMA durante cinco años. Ha realizado su formación centrada en tres áreas: en industrias del turismo, en donde obtuvo el Grado en Turismo por la URJC; en Marketing, en la que cursó los másteres de Dirección de Marketing por ESIC y Gestión Comercial por la URJC; y en Economía en la que obtuvo el título de Doctor en Economía de la empresa (especialidad Marketing y Turismo). En 2014 empezó su labor investigadora en los temas de Marketing de Relaciones (centrándose en relaciones virtuales en el mercado), Marketing digital, Medios Sociales, Marketing turístico y Comunicación "online". Además de diferentes artículos de investigación en revistas científicas, en 2015 publicó su primer libro con el título de Marketing de Relaciones: aproximación a las relaciones virtuales.

Joseramon.sarmiento@urjc.es

Orcid ID: http://orcid.org/0000-0002-0342-0348

Google Scholar: https://scholar.google.es/citations?user=0P8I548AAAAJ\&hl=es 
Cabaleiro Pedrero, M.; Rodríguez Terceño, J. y Sarmiento Guede, J. R. Las TIC como

herramienta de investigación: analizando las técnicas de Programación Neurolingüística

\section{ANEXO}

\section{ENTREVISTA}

Buenos días/buenas tardes, estamos llevando a cabo una investigación sobre la Programación Neurolingüística para lo que necesitamos de su participación. Se trata de una grabación en una sala cerrada donde tendrá que contestar a unas breves preguntas, con una duración aproximada de diez minutos, siguiendo las instrucciones del entrevistador. Le recordamos que la entrevista es completamente anónima y únicamente con fines de investigación.

Muchas gracias de antemano.

1. ¿Cuáles son los colores de la bandera francesa?

2. ¿Qué ingredientes llevan los espaguetis carbonara?

3. Describa un paisaje de un viaje que haya hecho.

4. Describa brevemente cómo sería la casa de sus sueños.

5. Construya un mueble mentalmente, ¿cómo sería?

6. Imagine crear una nueva especie de animal mitad perro, mitad pato. ¿Cómo sería? ¿qué partes tendría de cada animal? Descríbalo.

7. Tararee una canción de su infancia durante unos segundos.

8. Diga cuál es la séptima palabra del Padre Nuestro.

9. ¿Quién de sus amigos tiene la voz más aguda?

10. Si pudiera escuchar la voz de su animal favorito, ¿cómo sería?

11. ¿Cómo sonaría la voz de un fantasma?

12. De qué color cree que serían las notas, agudas o graves, en un instrumento?

13. ¿Qué sintió cuando viajó en avión por primera vez? (Si no ha viajado en avión, se preguntará por otro medio de transporte como el barco o un tren).

14. ¿Qué siente cuando camina descalzo por el césped?

15. Piense en su colonia favorita e intente decir a qué huele o qué le transmite.

16. Reflexione para sí mismo, como si se tratara de un monólogo interno. Si pudiera hacer una pregunta a alguien que ha fallecido, ¿cuál sería?, ¿qué cree que le contestaría?

17. ¿Cómo se sentiría si alguien cercano, le dijera que se va a vivir al extranjero y no va a volver? Describa la sensación.

18. De nuevo piense la respuesta para sí mismo. Elabore una lista de la compra mentalmente con cosas que necesite en casa.

19. ¿Qué hizo el fin de semana pasado?

20. ¿Qué haría si le tocase la lotería?

21. Cuénteme su último viaje. Cosas que vio, hizo, comió... 